\section{Archeologia dell'architettura e storia del territorio: la valle del Lucido (MS)}

SONIA GOBBATO

\section{Riassunto}

Questo studio si propone di interpretare storicamente i rusultati dell'analisi dell'edilizia dei centri abitati che conformano l'insediamento della valle del Lucido in Lunigiana (MS). La diacronia dell'architettura conservata e le tipologie edilizie, confrontate con i dati di scavo archeologico e le fonti scritte, permettono infatti di ripercorrere le tappe fondamentali dell'insediamento della valle e la sua articolazione, con una particolare attenzione ai fenomeni socioeconomici, per un periodo compreso tra I'XI e il XX secolo.

Parole chiavi: Storia dell'insediamento, edilizia storica, cronotipologia, tipologia edilizia.

\footnotetext{
Abstract

This paper presents the results of research in the valley of the river Lucido in the province of Massa-Carrara (MS), from the point of view of extensive analysis of architecture in historic centres. Through the detailed archaeological study of each of the constructions that make up these historic centres, using additional information obtained from archaeological excavations and written sources, a diachronic analysis can be made of the social history of the settling of the valley between the 11 th and 20th centuries. Specific attention is also paid to configuration processes in the village network, their transformation in the Middle Ages and their later transformation in the modern era.
}

Key words: Settlement history, architectonic typology, historic architecture, chronotypology.

\section{INTRODUZIONE ${ }^{1}$}

La ricerca realizzata nella valle del Lucido si pone come complemento degli studi sull'insediamento medievale e postmedievale svolti da Isabella Ferrando ed Elisabetta Crusi negli anni settanta, in valli limitrofe, dove si sono definite le prime cronotipologie delle aperture e delle tecniche costruttive, e per verificare la validità, dopo più di venti anni, dei risultati allora ottenuti (FERRANDO CABONA, CRUSI, 1979, 1981, 1988).

I principali limiti della lettura dell'edilizia storica in Lunigiana, già evidenziati dalle ricerche precedenti, sono dovuti all'assenza di strutture in elevato con fasi anteriori al trecento, tranne nel caso particolare degli edifici ecclesiastici, per i quali riusciamo a risalire all'XI e al XII secolo. Per questo motivo la lettura archeologica dell'architettura permette di realizzare una ricostruzione del fenomeno insediativo del territorio a partire dal bassomedioevo, lasciando per i periodi anteriori, e soprattutto per l'altomedioevo, solo delle ipotesi, che devono essere verificate attraverso i dati delle fonti scritte e mirate indagini archeologiche del sottosuolo.

Attraverso quindi l'integrazione di questi dati, quando disponibili, nella ricerca si è potuto delineare l'evoluzione dell'insediamento nella valle del Lucido, con una particolare attenzione allo sviluppo socioeconomico dei vari centri storici.

Si presentano in questa sede alcuni dei risultati ottenuti dall'applicazione della lettura archeologica dell'edilizia storica per lo studio dell'insediamento.

Il territorio scelto per questa analisi, la valle del torrente Lucido, è una valle trasversale del fiume Aulella, situata nella parte sud orientale della Lunigiana (fig. 1). Si tratta di un territorio molto particolare, che presenta in poco più di $14 \mathrm{~km}$. di lunghezza, un'estrema varietà di paesaggio, passando da un fondovalle pianeggiante, coltivato a grano e a orti, ai pascoli sopra i 1000 m. s.l.m. delle Alpi Apuane.

\section{METODOLOGIA}

Lo studio dell'architettura nella valle del Lucido ha previsto una ricognizione dell'edilizia storica in tutti i centri della valle, utilizzando come base diversi tipi di cartografie catastali, tra cui le carte vettoriali, realizzate recentemente dalla Regione Toscana con l'aereofotogrammetria. Particolarmente utili sono risultate inoltre le mappe del catasto

\footnotetext{
1 Desidero dedicare questo breve contributo alla memoria di Isabella Ferrando, che insieme a Tiziano Mannoni, mi ha ispirato e guidato nella realizzazione di questa ricerca.
} 


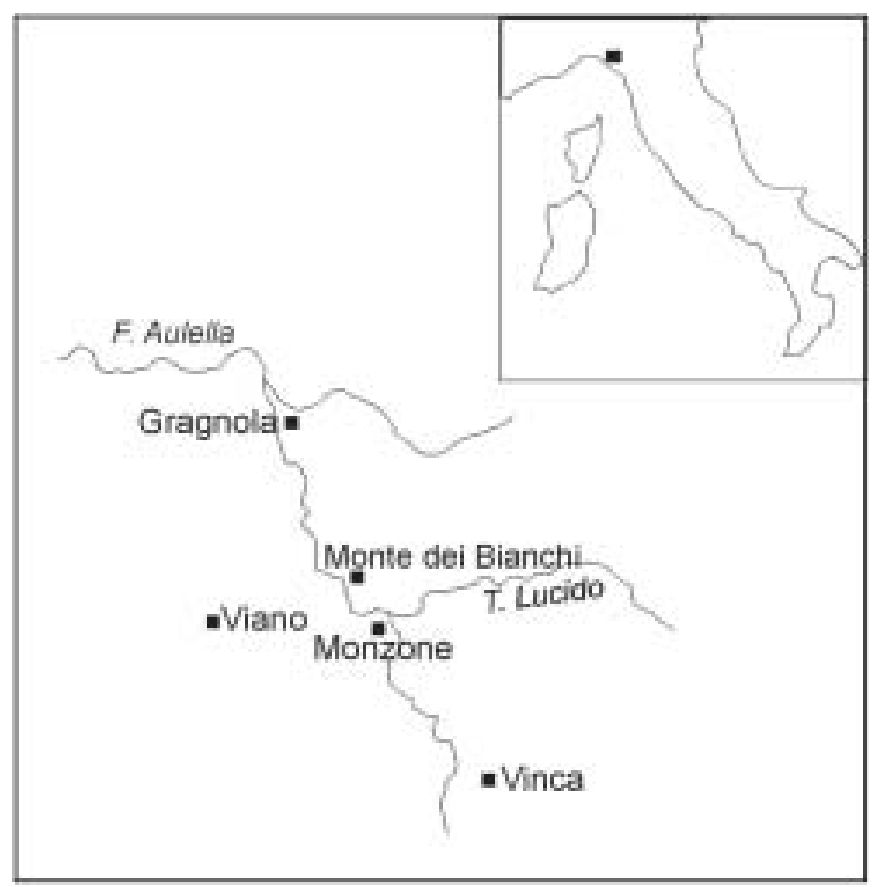

Fig. 1. Localizzazione della valle del Lucido in Italia

Leopoldino, realizzate negli anni venti del XIX secolo, su commissione del granduca Pietro Leopoldo, che riflettono la situazione urbanistica dei centri storici prima delle importanti trasformazioni documentate nel corso dell'ottocento e del novecento.

La valle del Lucido presenta attualmente venti centri abitati, tutti di fondazione almeno medievale, concentrati soprattutto sulle sponde e sulle colline che costeggiano il torrente Lucido e la sua diramazione verso Equi. Applicando la metodologia utilizzata nelle ricognizioni archeologiche, è stata realizzata una prima indagine preliminare al fine di determinare i centri campioni in cui realizzare una ricerca approfondita. L'indagine preliminare ha previsto uno spoglio della bibliografia riguardante il territorio, che in questo caso è costituita essenzialmente dagli studi degli storici locali, sulle fonti scritte e dei dati provenienti dagli scavi archeologici realizzati nella Tecchia di Equi.

A questa prima fase è seguita una prima ricognizione generale in tutti i centri della valle, per raccogliere $i$ dati sufficienti a definirli tipologicamente e verificare le potenzialità archeologiche dell'edilizia storica. Le tipologie individuate, anche se non applicabili per tutti i centri, sono i borghi fortificati d'altura, le ville aperte, le pievi e i centri mercantili di fondovalle. Si tratta di tipologie molto generiche che presentano ancora molti limiti di definizione. Alcuni centri infatti possono appartenere a più tipologie o aver cambiato nel corso del tempo le proprie caratteristiche socioeconomiche e quindi urbanistiche, e ci sono invece centri che non si riescono ad inserire in nessuna di queste classi. Questa indagine preliminare ha evidenziato la ricchezza architettonica della maggior parte dei centri della valle del Lucido, e la scelta del campione è stata quindi determinata dalla tipologia insediativa e anche dal ruolo politico e sociale dell'abitato nel corso dei secoli. I centri prescelti per un'analisi estensiva sono Gragnola, Viano e Monte dei Bianchi.

Per ognuno di questi centri sono stati raccolti i dati relativi alle tipologie edilizie, alle tecniche costruttive e alle tipologie di aperture di ogni edificio, oltre a determinare, dove possibile, le principali fasi cronologiche delle varie unità edilizie (CUCINI et alii, 1988; FERRANDO, 1990).

I dati raccolti in una scheda appositamente formulata, sono stati quindi utilizzati per creare una piattaforma Gis, con un programma di gestione territoriale, e poter quindi visualizzare i risultati della ricerca attraverso carte tematiche (BIANCHI, NARDINI, 2000).

\section{I CENTRI STORICI}

I centri scelti per l'analisi a tappeto, Gragnola, Viano e Monte dei Bianchi rappresentano rispettivamente il centro commerciale, il centro religioso-monastico e la sede del potere feudale nel corso del medioevo. Naturalmente il loro ruolo ha cambiato anche notevolmente nel corso dei secoli, ma questa particolare valenza politica e sociale ha determinato in modo rilevante il loro aspetto architettonico e urbanistico che conservano ancora oggi.

\section{Gragnola}

Il primo borgo che si incontra entrando nella valle del $\mathrm{Lu}$ cido dalla valle Aulella è Gragnola. Posto a circa $160 \mathrm{~m}$. s.l.m., il centro si sviluppa in una fascia pianeggiante che si estende tra l'estremità montuosa dove sorge il castel dell'Aquila e il torrente Lucido. Il centro storico si presenta a pianta quadrangolare, organizzato lungo la via detta di Borgo Lucido, la via $S$. Nicola e intorno alla piazza centrale, antica piazza del mercato (fig. 4). Una parte di più recente formazione si è sviluppata al di la del torrente, lungo le strade per Colla e per Cortila, dove si trova anche il cimitero. Questa zona pianeggiante si caratterizza per la presenza di orti e campi coltivati, aspetto che doveva avere anche nel medioevo.

Documentato per la prima volta nel 1162, Gragnola diventa agli inizi del XIII secolo sede podestarile, rivestendo fino al quattrocento un rilevante ruolo politico ed economico all'interno della valle (LUPO GENTILE, 1912).

La progressiva perdita di importanza di questo centro nel corso del postmedioevo, ha permesso la conservazione eccezionale di alcune tipologie edilizie, sconosciute in altri 


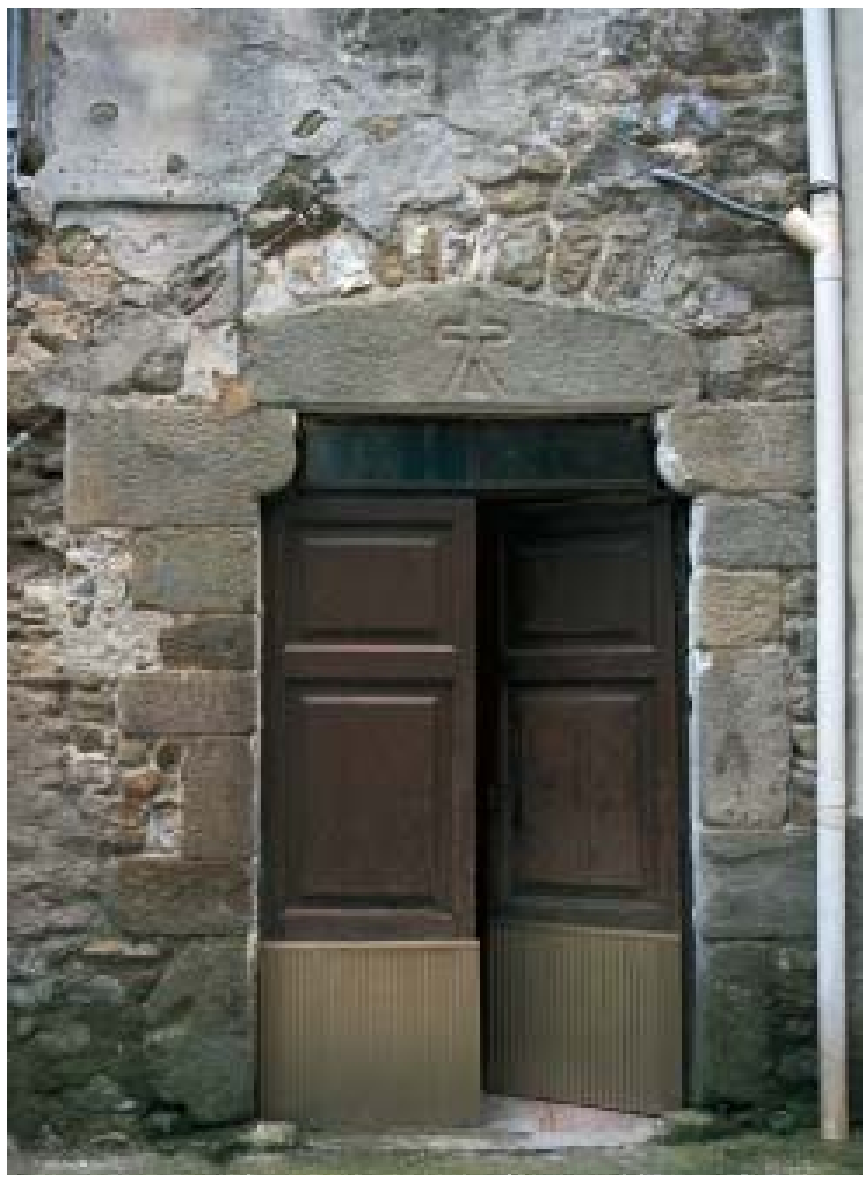

Fig. 2. Portale del xv secolo riutilizzato in una casa postmedievale di Gragnola (MS)

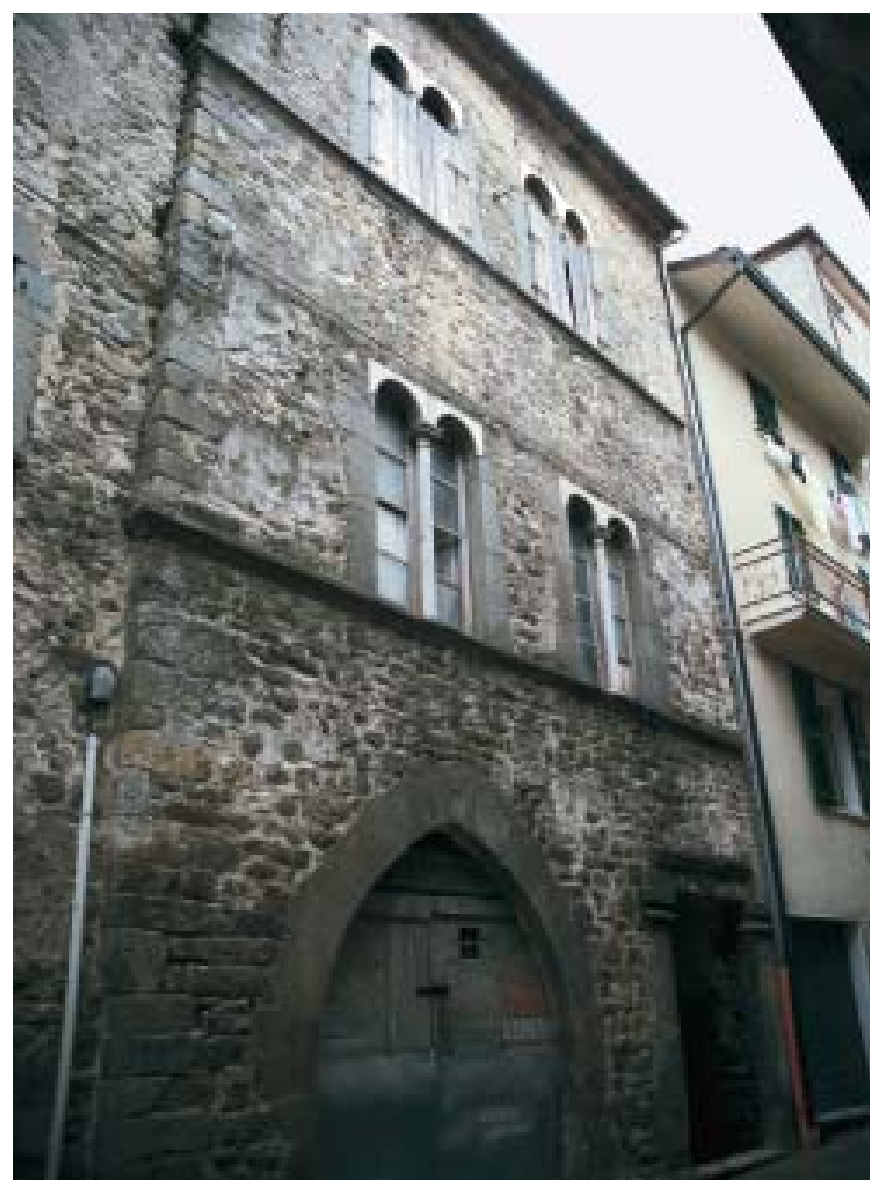

Fig. 3. Palazzo-bottega di Gragnola

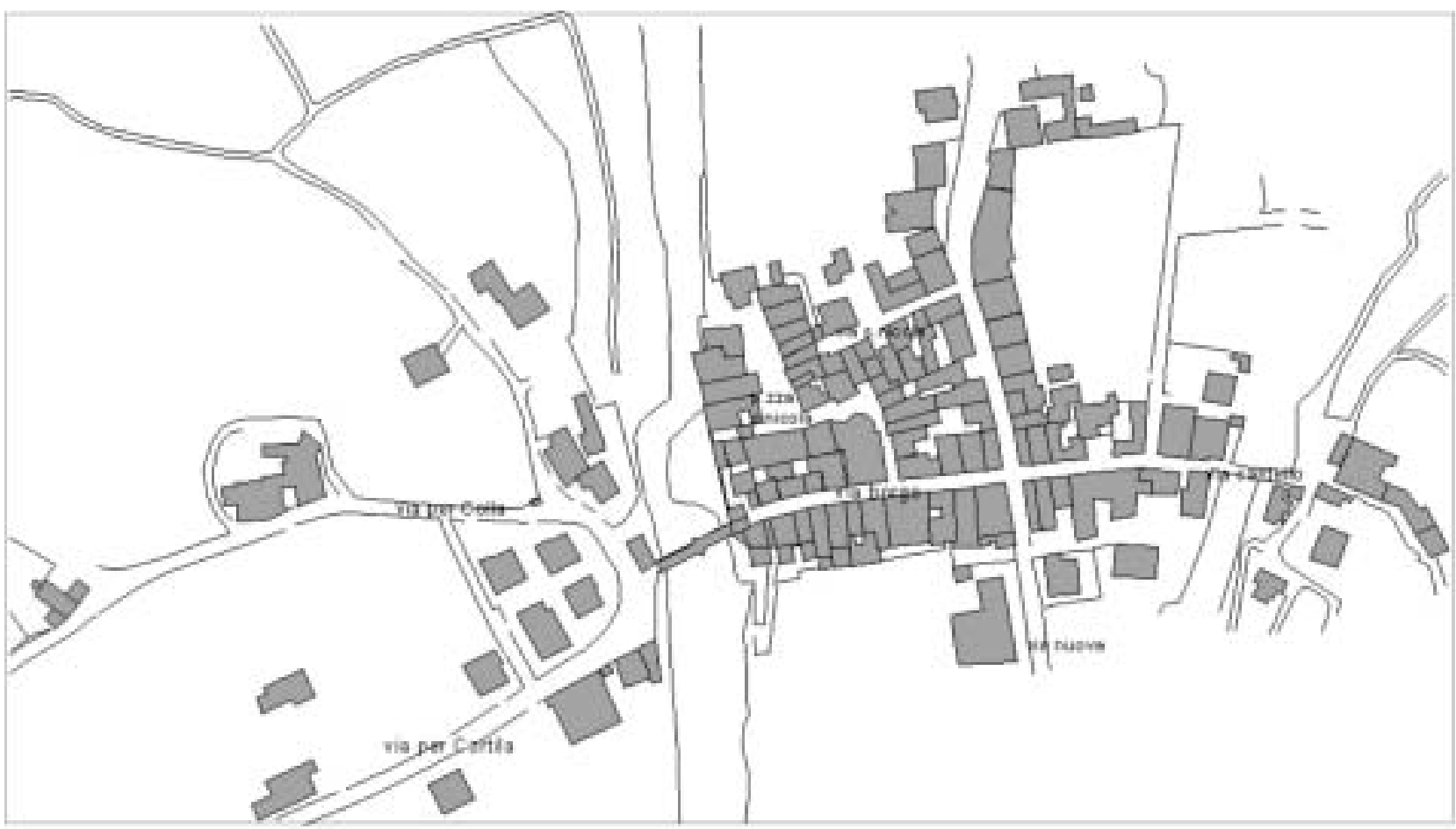

Fig. 4. Planimetria di Gragnola (MS) 
settori della Lunigiana, anche se in quasi totale abbandono. Il suo carattere fortemente commerciale è, infatti, evidenziato da una particolare concentrazione di case-botteghe, databili dal XIV al XVIII secolo, tra cui emerge un tipo di edificio che si è deciso di ridefinire come "palazzo-bottega", e che si afferma tra il tre e il quattrocento (fig. 3). Si tratta di una struttura commerciale e residenziale, che si distingue per la particolare ricercatezza architettonica e decorativa, come la presenza di bifore nei piani alti, portali scolpiti, e anche per le sue dimensioni, che trovano paralleli solo in ambito cittadino.

Nonostante l'indagine svolta non possa considerarsi esaustiva, i dati raccolti ci permettono di ricostruire nelle sue fasi fondamentali lo sviluppo urbanistico e architettonico di Gragnola dal XV al Xx secolo. Il maggior limite della ricerca risiede proprio nella quasi totale assenza di elementi anteriori al quattrocento, periodo nel quale conosciamo l'importanza politica e commerciale del centro, ma ne ignoriamo le forme urbanistiche. Gli unici edifici che si possono infatti collocare tra il XII secolo, data della prima citazione di Gragnola nelle fonti scritte, e il quattrocento sono il ponte sul torrente Lucido e la cappella, che probabilmente presiedeva alla sua manutenzione. La presenza del ponte testimonia e conferma il forte legame di Gragnola con la viabilità.

Il XV secolo si caratterizza per una generale e radicale ricostruzione del borgo (fig. 2). Le nuove tipologie e tecniche costruttive, si diffondono rapidamente non solo a Gragnola ma in tutto il territorio e nelle valli limitrofe. Questo fenomeno testimonia sicuramente una consolidata ricchezza e sicurezza politica, che in Gragnola si realizza soprattutto attraverso il commercio e il suo ruolo di capoluogo di riferimento per il feudo di appartenenza. La ricchezza evidenziata nei palazzi con botteghe costruiti in Gragnola nel XV secolo, è sicuramente la migliore testimonianza di un nuovo ceto sociale di commercianti, che raggiunge il suo culmine proprio in questo secolo.

Per quanto riguarda l'estensione del borgo i dati raccolti permettono di ipotizzare che avesse le dimensioni del centro storico attuale, articolato tra la via Nuova e il fiume. La regolarità e la particolare forma quadrangolare del centro storico si sono conservate probabilmente per l'esistenza di una cinta muraria, non più leggibile, ma documentata come ancora esistente nel XVII secolo.

Nel corso del XVI e XVII secolo non si osservano interventi importanti nell'edilizia di Gragnola, che probabilmente mantiene quasi inalterate le forme quattrocentesche, con modifiche strutturali non radicali.

Una vera rivoluzione edilizia inizia nel corso del XVIII per concludersi agli inizi del XX secolo. In questo periodo compaiono, infatti, grandi case, frutto spesso dell'accorpamento e ristrutturazione di edifici preesistenti che riflettono un cambio importante nell'articolazione sociale del borgo. Questo fenomeno, che è comune a tutti i centri non solo della valle del Lucido, e che meglio si può apprezzare negli insediamenti rurali, è legato all'affermarsi del sistema mezzadrile, che, anche se con un certo ritardo rispetto al resto della Toscana, si impone in queste aree periferiche (IMBERCIADORI, 1953). Ricchi imprenditori spesso provenienti dalle città come Lucca e Milano, investono nel territorio di Gragnola, accaparrandosi gran parte delle risorse agricole, ma a differenza di ciò che accadeva nel XV secolo, quando il proprietario manteneva comunque una elevata frammentazione nella coltivazione e il locatario era libero nelle gestione del terreno, ora il proprietario dirige direttamente lo sfruttamento dei propri terreni, su cui gli agricoltori locali lavorano come dipendenti (MARTINELLI, NUTI, 1974). Nel caso di Gragnola, che mantiene un ruolo commerciale e politico all'interno della valle, gli esponenti di questa classe sociale sono anche commercianti e rivestono le principali cariche pubbliche.

Segno della prosperità che vive Gragnola in questo periodo è la ricostruzione integrale della chiesa dei SS. Ippolito e Cassiano, che solitamente veniva realizzata con le forze della comunità. La continua crescita demografica fa sì che agli inizi del XIX secolo la chiesa debba essere nuovamente ampliata, questa volta con l'aiuto del granduca Francesco IV. Probabilmente l'ampliamento è reso possibile dall'abolizione, con la legislazione napoleonica, del cimitero presso la chiesa e il suo spostamento all'esterno del borgo. La recente perdita di importanza da parte di Gragnola, soprattutto a causa dell'accentramento amministrativo e commerciale di Fivizzano, capoluogo attuale del comune, ha protetto in parte l'edilizia del centro storico da ricostruzioni e restauri arbitrari. I primi segni di questa flessione si intravedono già nel XVI-XVII secolo, quando il centro rimane tagliato fuori dalla viabilità principale che si attesta tra la costa, Pontremoli, Fivizzano e la Pianura Padana (BARONI, 1998). Agli inizi del XIX secolo sappiamo dell'esistenza in Lunigiana di solo quattro centri in cui si realizzano mercati settimanali: Aulla, Pontremoli, Bagnone e Fivizzano (PeLLEGRINETTI, 1988: 150-152). La presenza di botteghe indica che Gragnola rimane comunque il mercato di riferimento per la valle, per cui svolge il ruolo di smistamento delle merci in transito.

\section{Viano}

Il castello di Viano sorge su di una altura a $498 \mathrm{~m}$. di altitudine, in posizione dominante sulla bassa valle del Lucido (fig. 5). Il centro attuale è collegato con la viabilità di fon- 
dovalle dalla strada carrozzabile, ma si conservano due mulattiere di cui una a mezza costa raggiunge gli abitati che si dispongono sul versante sinistro della valle fino a Gragnola, e l'altra lo collega a Cecina nella valle del Bardine. Si tratta probabilmente di una viabilità antica, di tipo locale, a cui si è preferita in tempi recenti la più comoda strada di fondovalle per Gragnola e la via che attraversa Tenerano e Villa, per la valle del Bardine.

Sebbene ci siano vari riferimenti a questo centro nella documentazione scritta nel corso del 1100, Viano assume un ruolo politico importante solo a partire dalla metà del XIII secolo, quando diventa la sede del ramo superstite dei Bianchi, a seguito dell'acquisizione del resto del territorio da parte dei Malaspina, che lo conquistano nel 1352. Nel corso del XVI secolo Viano diventa la sede di un feudo autonomo assegnato ad un ramo della famiglia Malaspina. Nel corso del postmedioevo la sua importana decade, trasformandolo in un piccolo borgo contadino, dove le stesse torri e mura si convertono in stalle e fienili.

Il castello di Viano presenta una pianta quasi ellissoidale, determinata dalla cinta muraria che lo circonda interamente e che segue la forma del colle su cui il castello si è sviluppato. L'abitato non ha occupato in realtà l'intero colle, dato che le mura ne escludono la parte più occidentale, sul cui lato sono posti gli unici due accessi. Anche al suo interno l'abitato ha seguito i dislivelli naturali del terreno sviluppandosi su quattro fasce quasi concentriche, di cui la più esterna termina nelle due porte del castello.

Nonostante le trasformazioni postmedievali Viano conserva in gran parte l'aspetto fortificato medievale, con ampi tratti della cinta muraria, cinque torri e le due porte d'accesso al castello (fig. 6).

L'analisi dell'edilizia del castello ha permesso di evidenziare tre grandi fasi, corrispondenti ad altrettanti ristrutturazioni generali del centro. Particolarmente importante è la prima di queste fasi che rappresenta il momento di riorganizzazione del castello con la ricostruzione delle mura nel XIV secolo. Quest'opera imponente e molto omogenea, realizzata probabilmente nel momento del passaggio del castello ai Malaspina, si conserva ancora quasi nella sua totalità e ha sicuramente determinato una ristrutturazione anche all'interno del borgo, come testimoniano alcuni portali conservati.

Per quanto riguarda il XV e il XVI secolo i manufatti individuati sono troppo esigui per permetterci di definire una o più tipologie edilizie che non sia quella della semplice abitazione. Interessante è comunque l'assenza di strutture mercantili. Diffuse erano sicuramente anche le stalle, che come oggi si distribuivano nel borgo intorno all'area residenziale con accesso alla viabilità lungo le mura. L'alleva-

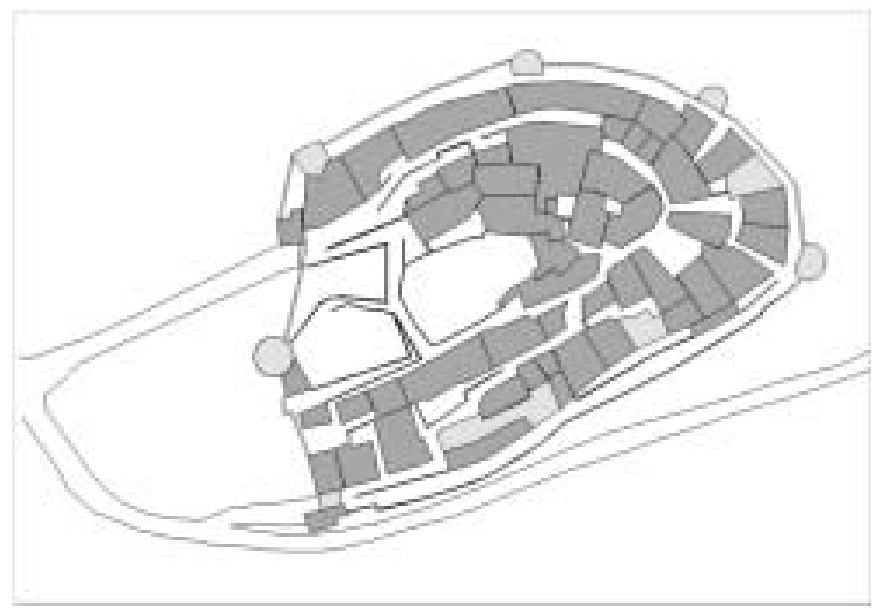

Fig. 5. Planimetria di Viano (MS)a

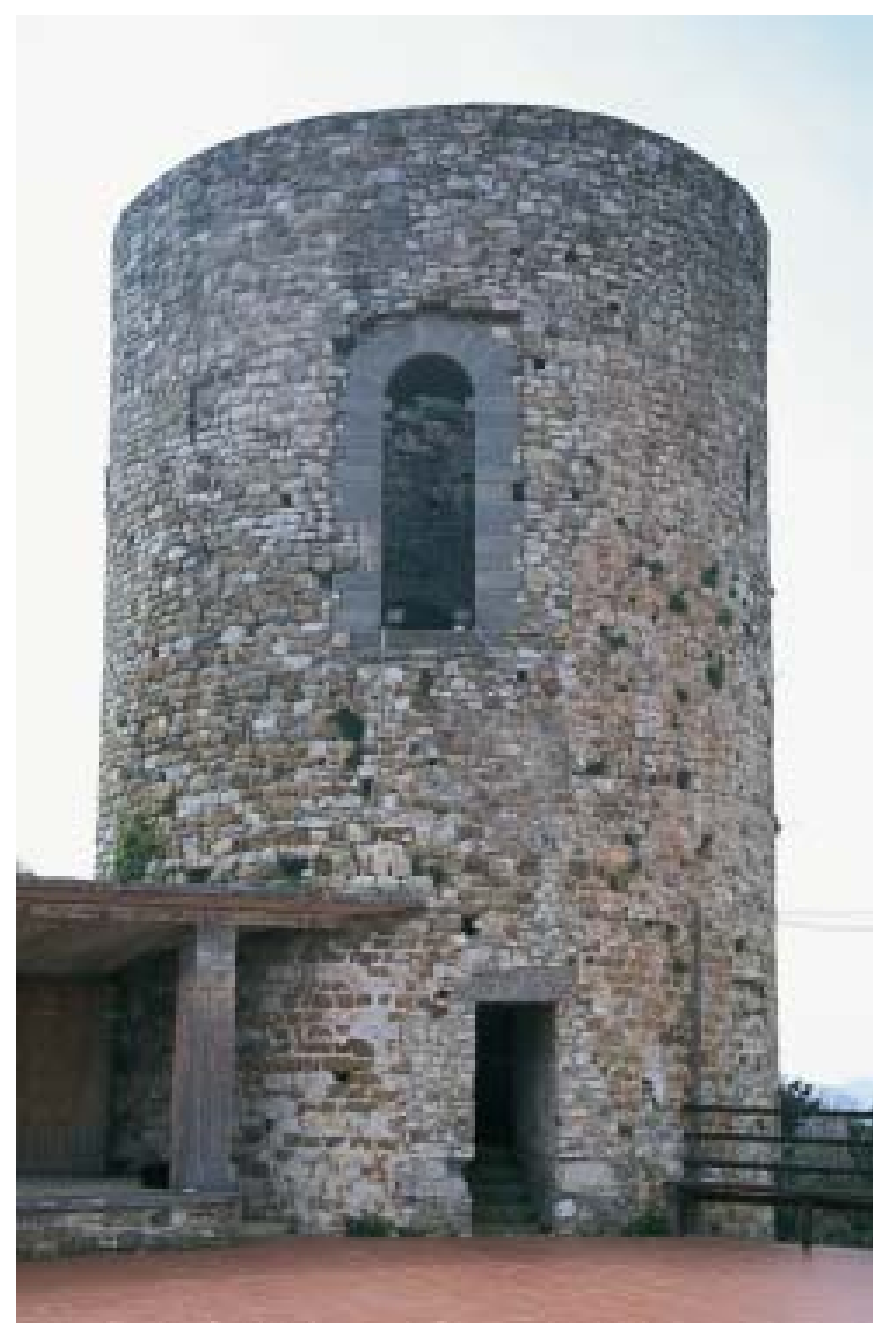

Fig. 6. Torre dell'area signorile di Viano (MS) 
mento di pecore e maiali, testimoniato fino a qualche decennio fa, rispecchia probabilmente una situazione analoga al tardomedioevo, soprattutto in questa parte della valle, esposta a nord e che favorisce quindi l'allevamento di bestiame e la coltura delle castagne. Questo tipo di economia si è mantenuta inalterata fino alla metà del XX secolo, quando, a seguito della massiva emigrazione, le attività agricole vengono progressivamente abbandonate. Il seicento è invece un secolo di rivoluzione edilizia, sebbene anche in questo caso i manufatti di cui disponiamo si trovano spesso fuori contesto. Le fonti non ci consentono di collegare questa fase con sviluppi politici o economici particolari, ma le fonti scritte ci confermano che la rocca del marchese era in corso di costruzione, o si era interrotta. Se veramente in questo periodo è avvenuta una ristrutturazione della parte residenziale, forse collegata con il ritorno del centro al ramo dei Malaspina di Fosdinovo, è probabile che l'attività di muratori e scalpellini all'interno del castello abbia offerto l'opportunità di una ristrutturazione anche ad altre case del borgo. A differenza di Gragnola e di Monte dei Bianchi non si osserva in questo centro la presenza di un rinnovamento ottocentesco con l'arrivo di capitali dall'esterno, probabilmente per la posizione marginale rispetto alla viabilitá e la sfavorevole esposizione del suo territorio, occupato principalmente da boschi. Si osserva invece negli ultimi secoli una riduzione dell'abitato, con l'abbandono di diversi edifici, il crollo e scomparsa delle strutture residenziali fortificate e per questo motivo il borgo riesce a mantenere praticamente intatto il circuito murario.

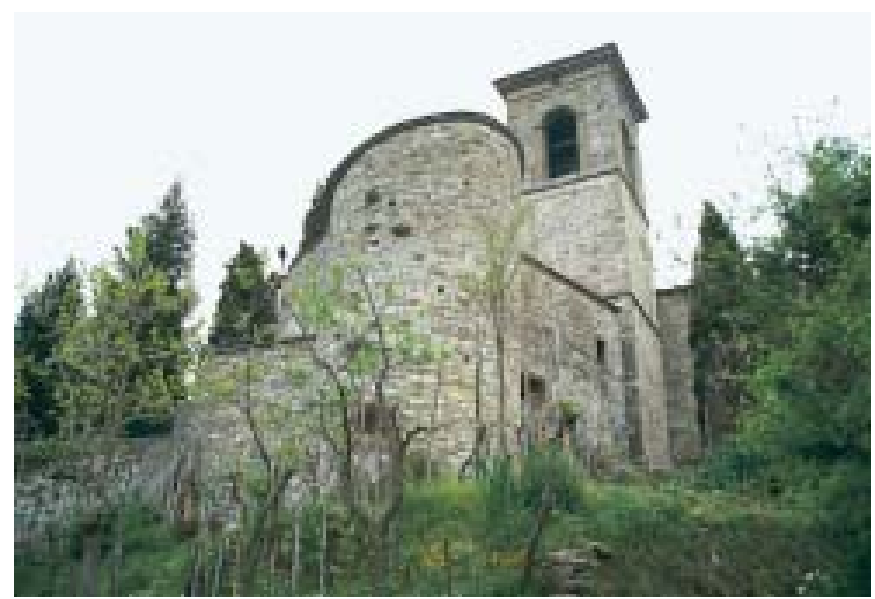

Fig. 7. La cinta muraria di Monte dei Bianchi vista da sud-est

\section{Monte dei Bianchi}

Monte dei Bianchi sorge su un promontorio sul versante destro del torrente Lucido, in posizione dominante rispetto al fondovalle. Il centro si forma probabilmente intorno al monastero di San Michele Arcangelo, che sappiamo nell'XI secolo essere cenobio dei Bianchi d'Herberia, signori locali (BARONI, 1989-1991). Dell'antico monastero rimane solo la chiesa, che conserva nel lato nord i resti della sua prima fase costruttiva, databile tra l'XI e il XII secolo, realizzata con bozze di calcare bianco disposte su filari orizzontali e paralleli. Si tratta del paramento murario più antico, finora conservato in elevato nella valle.

Il borgo di Monte dei Bianchi presenta una pianta dalla forma approssimativamente rettangolare, che si sviluppa lungo un crinale, attraversato da una via centrale, alla fine della quale, sul lato ovest, sorge la chiesa e uno spazio aperto all'interno delle mura, dove era in origine il monastero di S. Michele Arcangelo (fig. 9). La cinta muraria che circondava il borgo è riconoscibile sono in alcuni punti e aveva tre accessi di cui uno all'estremità ovest della via centrale e due laterali, oltre a diverse torri, di cui si conservano solo quattro sul versante sud (fig. 7). L'impianto fortificato si data per analogie della morfologia e delle tecniche costruttive al XIV secolo, coetaneo alla maggior parte delle cinte murarie di questa parte della Lunigiana. La particolaritá di Monte dei Bianchi è però l'assetto urbanistico che sembra pianificato nel momento della sua fortificazione, lasciando all'esterno di questo progetto solo l'area del monastero con la chiesa, che effettivamente erano separate dal borgo da una porta interna alla cinta. Il confronto con altri borghi con una maggiore documentazione scritta ci permette di affermare che Monte dei Bianchi viene rifondato proprio nel corso del XIV secolo. Queste nuove fondazioni o rifondazioni sono legate ad un fenomeno più chiaramente riscontrabile in Versilia e nella pianura del Valdarno, dove si diffondono borghi nuovi o la fortificazione di centri preesistenti lungo i confini politici per rinforzarli. Monte dei Bianchi nonostante alterne vicende si colloca nel tardo medioevo proprio sul confine tra feudi, anche se la mancanza di fonti scritte ci impedisce di determinare il fautore di questa rifondazione.

Le trasformazioni postmedievali sono state molto pesanti e hanno provocato la quasi totale scomparsa delle strutture medievali. Ciononostante, $i$ resti individuati in prossimità della chiesa e nel borgo sembrano testimoniare la presenza di case di una certa dimensione e con elementi architettonici rilevanti. Come in altri centri della valle il XVIII secolo rappresenta un periodo di rivoluzione edilizia determinata soprattutto dall'affermarsi di nuove famiglie di ricchi proprietari terrieri che comprano e ristrutturano 


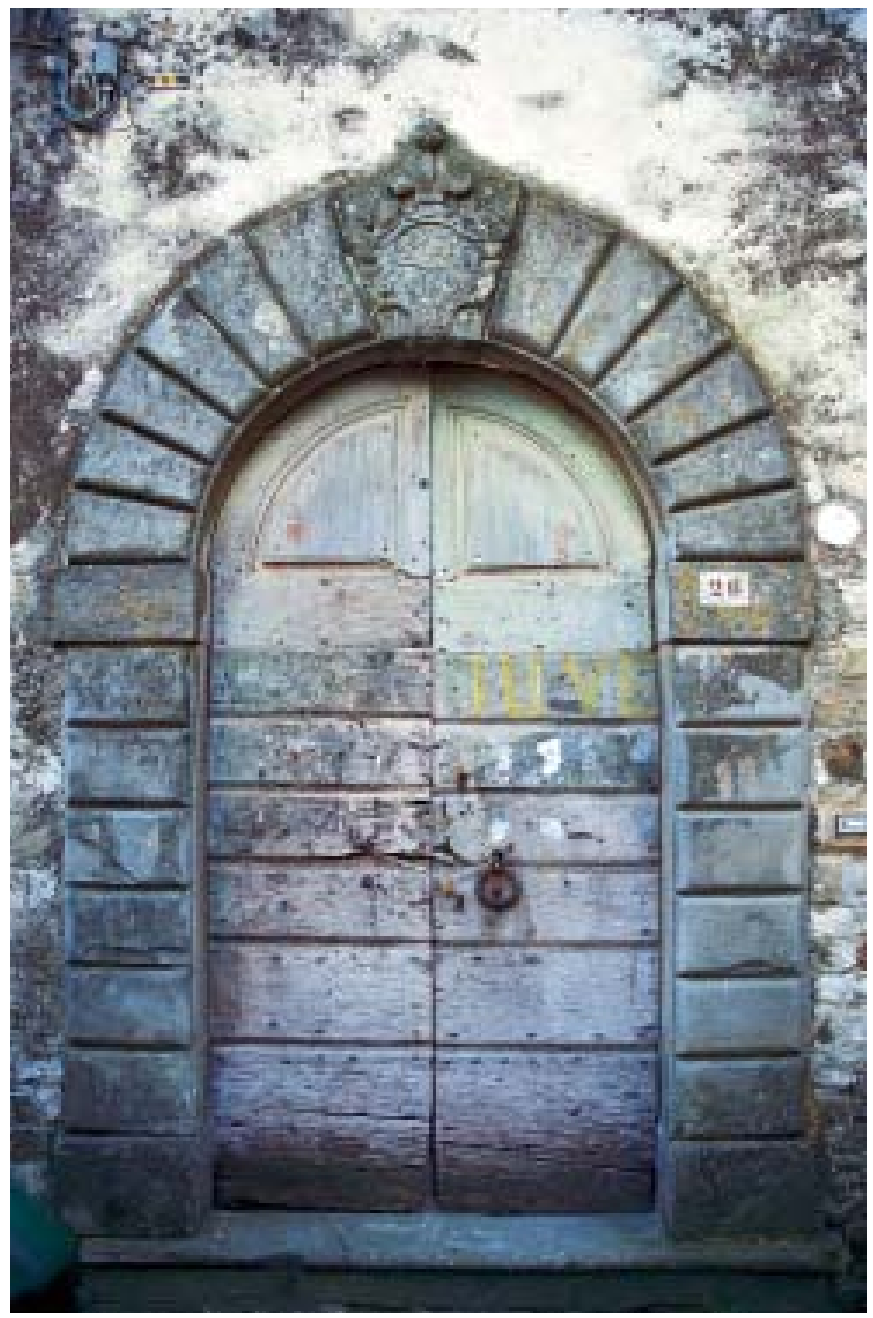

Fig. 8. Portale settecentesco di un palazzo di Monte dei Bianchi (MS)

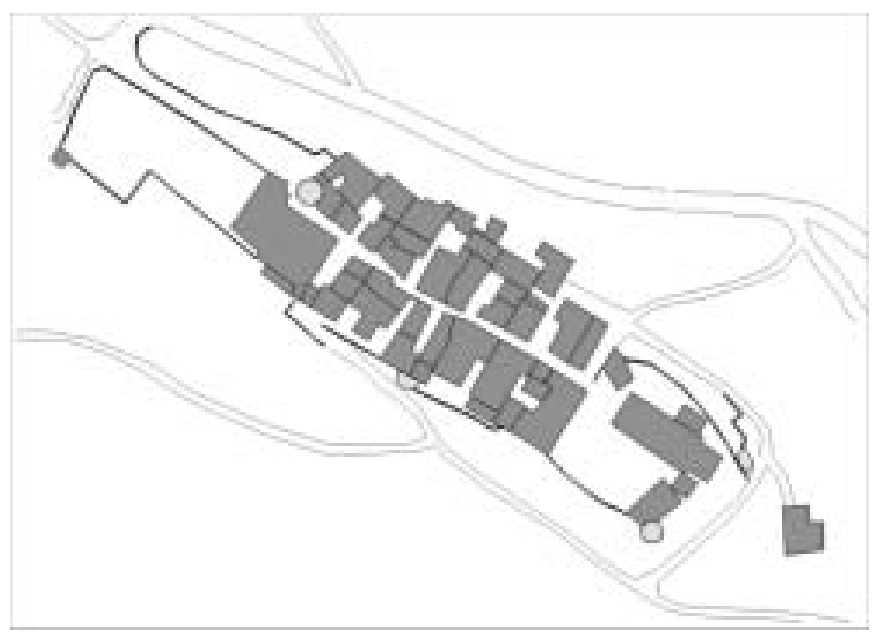

Fig. 9. Planimetria di Monte dei Bianchi (MS) gli edifici medievali (fig. 8). Nel caso di Monte dei Bianchi questo fenomeno si manifesta in un più generale rinnovamento del borgo e nella ristrutturazione della chiesa. In questa fase il borgo comincia anche ad espandersi, raggiungendo e inglobando in più punti il circuito murario. L'ultima importante fase edilizia è quella ottocentesca in cui si conferma l'importanza di alcune famiglie che ampliano i propri palazzi. Il borgo presenta comunque fin dal medioevo uno spiccato carattere agricolo, favorito dall'esposizione al sud di gran parte del suo territorio, dove ancora si conservano orti e uliveti oltre a tre stalle ancora attive.

\section{L'INSEDIAMENTO NELLA VALLE DEL LUCIDO}

La ricognizione dell'architettura dei centri storici della valle del Lucido ha fornito dati molto interessanti per capire alcuni fenomeni socioeconomici che hanno caratterizzato questo territorio nel corso del medioevo e del postmedioevo (MANNONI, 1993).

Il bassomedioevo si caratterizza per la presenza diffusa di castelli o villaggi fortificati, probabilmente frutto dell'accentramento dell'insediamento altomedievale, che ruotano intorno al centro amministrativo e commerciale di Gragnola, sede del mercato e della podesteria consortile dei signori locali, i Bianchi d'Herberia. Di questa fase si conservano essenzialmente le chiese, che vengono fondate o ricostruite tra l'XI e il XII secolo su commissione signorile, con tecniche regolari.

Tra il XIV e il XV secolo, con il dominio dei Malaspina, si osserva una rivoluzione edilizia, che comporta la quasi totale ricostruzione dei centri storici, e la scomparsa delle fasi costruttive anteriori. Del trecento sono infatti le cinte murarie e le torri conservate, rinnovate probabilmente con l'insediamento dei Malaspina nei castelli della valle. Quattrocentesca è invece quasi tutta l'architettura civile medievale conservata, con esempi interessanti in quasi tutti i centri storici.

Con la divisione della valle tra la Signoria di Firenze e il marchesato dei Malaspina, avvenuta nel corso del XV secolo, si formano due tendenze opposte nell'evoluzione edilizia dei centri storici. Mentre gli abitati appartenenti al marchesato, conservano sostanzialmente fino al XIX secolo le dimensioni e l'aspetto raggiunti nel bassomedievo, nel resto della valle si afferma, nel corso del postmedioevo, una nuova classe sociale, formata da grandi proprietari terrieri provenienti dall'esterno, che oltre a determinare la crescita economica e urbanistica dei singoli centri, li trasforma architettonicamente con la costruzione di grandi case padronali, associate a cappelle private e ad una serie di edifici di servizio. 
Un ultimo importante fenomeno edilizio si osserva nel corso del XIX secolo, con la fine del marchesato e l'inizio dell'attività di estrazione e lavorazione del marmo del monte Sagro. L'apertura delle cave porta a un breve periodo di floridezza economica, durante il quale avviene la maggiore espansione urbanistica dei centri abitati e la trasformazione di gran parte dell'architettura civile.

\section{Bibliografia}

BARONI F., 1989-1991, Sulla fondazione del monastero di San Michele Arcangelo di Monte dei Bianchi, Cronaca e Storia della Val di Magra, XVIII-XX, pp. 77-89.

BARONI F., 1998, Rapporti e collegamenti viarii medievali attraverso il passo di Tea fra la Garfagnana, la Lunigiana e il mare, La Garfagnana dall'epoca comunale all'avvento degli Estensi, Modena, pp. 163-210.

BiAnCHI G., NARDini A., 2000, Archeologia dell'architettura di un centro storico. Proposta per un'elaborazione informatica dei dati su piattaforma G.I.S. bidimensionale, in atti del II Congresso Nazionale di Archeologia Medievale, Brescia, pp. 381-388.

BrANCHI E., 1898, Storia della Lunigiana feudale, Bologna.

Cucini C., Guideri S., PaOlucci G., Valenti M., 1988, Proposte per una lettura ragionata delle emergenze in elevato e in superficie sul territorio, in M. PASQUinuCCI E S. MenChelli (a cura di), La cartografia archeologica, Pisa, pp. 53-102.

FERRANDO I., 1990, Archeologia dell'edilizia povera in Lunigiana. Metodi e primi risultati, in G. L. MAFFEI (a cura di), La casa rurale in Lunigiana, Venezia, pp. 151-166.

Ferrando Cabona I., Crusi E., 1979, Archeologia del territorio: proposta metodologica sull'esempio dello Zignago (Zignago 2), Archeologia Medievale, VII, pp. 247-270.

Ferrando Cabona I., Crusi E., 1981, Storia dellinsediamento in Lunigiana: valle del Rosaro, Genova.

Ferrando Cabona I., Crusi E., 1988, Storia dellinsediamento in Lunigiana: Alta Valle Aulella, Genova, $2^{\circ}$ ed.

Ferrando I., Mannoni T., Pagella R., 1989, Cronotipologia, Archeologia Medievale, XVI, pp. 647-661.

GALLO N., 1993-1994, Insediamenti, architettura e tecniche costruttive della Lunigiana medievale, tesi di laurea inedita, Università degli Studi di Firenze, Firenze.
Gobвato S., 2001, Storia dell'insediamento in Lunigiana: la valle del Lucido, tesi di specializzazione in archeologia medievale inedita, Università di Pisa.

Gobbato S., Quirós Castillo J.A., 2004, Prospección y Arqueología de la Arquitectura, Arqueología Espacial 22 (en prensa).

IMBERCIADORI I., 1953, Campagna toscana nel '700, dalla reggenza alla restaurazione 1737-1815, Firenze.

Lupo Gentile M., 1912, Il regesto del Codice Pelavicino, Società Ligure di Storia Patria, vol. XLIV, Genova.

Mannoni T., 1984, Metodi di datazione dell'edilizia storica, Archeologia Medievale, XI, pp. 396-403.

MANNONI T., 1993, Modi di fare storia con l'archeologia. Variazione su un tema di Andrea Carandini, Archeologia Medievale, XX, pp. 561-568.

Mannoni T., 1994, Caratteri costruttivi dell'edilizia storica, vol. 3 della collana Venticinque anni di archeologia globale, Genova.

MANNONI T., 1994-95, Il metodo chiamato "archeologia globale" in Lunigiana, Memorie della Accademia Lunigianese di Scienze Giovanni Capellini, XIV-LXV, pp. 13-23.

Mannoni T., Cabona D., Ferrando I, 1988, Archeologia globale del territorio. Metodi e risultati di una nuova strategia della ricerca in Liguria, in Structures de l'habitat et occupation du sol dans les pays méditerranéens. les méthodes et l'apport de l'archéologie extensive, Parigi, pp. 43-58.

Martinelli R., Nuti L., 1974, Il territorio storico della Vicaria di Minucciano, La Provincia di Lucca, XIV/ 2, pp. 62-75.

NobiLi M., 1982, Famiglie signorili di Lunigiana fra vescovi e marchesi (secoli XII e XIII), in I ceti dirigenti dell'età comunale nei secoli XII e XIII, Pisa, pp. 233-265.

Nobili M., 1987-1988, Signorie e comunità nella Lunigiana orientale fra XI e XIII secolo, Memorie dell'Accademia Lunigianese di Scienze Giovanni Cappellini, vol. LVII-LVIII.

PANSINI G., 1976, Le comunità della Lunigiana e la riforma comunale di Pietro Leopoldo, Cronaca e Storia della Val di Magra, anno IV.

PellegrinetTi G., 1988, La Lunigiana e l'Impero Francese (1808-1814), Pontremoli.

Pellegrinetti G., 1993, La valle del Lucido nei secoli, Fivizzano

SETTiA A. A., 1985, La toponomastica come fonte per la storia del popolamento rurale, in V. FuMAGAlli e G. RossetTi (a cura di), Medioevo rurale. Sulle tracce della civiltà contadina, pp. 35-56.

WiCKHAM C., 1997, La montagna e la città. L'Appennino toscano nell'altomedioevo, Torino. 